\title{
Isolated Avulsion of the Common Hepatic Duct from Blunt Abdominal Trauma
}

\author{
Victor W. Wong, ${ }^{1}$ Arvin Gee, ${ }^{1,2}$ Paul Hansen, ${ }^{2,3,4}$ and Andrew Michaels ${ }^{1,2}$ \\ ${ }^{1}$ Department of Surgery, Oregon Health and Science University, 3181 SW Sam Jackson Park Road, Portland, OR 97239, USA \\ ${ }^{2}$ Department of Surgery, Legacy Emanuel Medical Center, 2801 N. Gantenbein, Portland, OR 97227, USA \\ ${ }^{3}$ Portland Providence Cancer Center, 4805 NE Glisan Street, Portland, OR 97213, USA \\ ${ }^{4}$ The Oregon Clinic, 4805 NE Glisan Street, Portland, OR 97213, USA
}

Correspondence should be addressed to Arvin Gee, arvin.gee@gmail.com

Received 15 May 2012; Accepted 12 June 2012

Academic Editors: C. Foroulis and M. L. Quek

Copyright ( 12012 Victor W. Wong et al. This is an open access article distributed under the Creative Commons Attribution License, which permits unrestricted use, distribution, and reproduction in any medium, provided the original work is properly cited.

\begin{abstract}
Isolated extrahepatic biliary tract injury following blunt abdominal trauma is rare. The underlying pathogenic mechanisms remain obscure, but include shear and/or compression forces on the biliary system. Associated morbidity rates are high and largely the result of delays in diagnosis. Imaging modalities commonly employed for diagnosis include ultrasonography, computed tomography, nuclear medicine, and magnetic resonance imaging. Percutaneous and endoscopic techniques have been used both for diagnosis and treatment. Treatment options are dictated by the stability of the patient and the extent of bile duct and concomitant injuries. In this paper, we discuss a case of isolated avulsion of the hepatic duct confluence following blunt trauma that was successfully managed with Roux-en-Y hepaticojejunostomy. To our knowledge, this specific injury pattern has not been previously reported.
\end{abstract}

\section{Introduction}

Isolated biliary tract injury following blunt abdominal injury is extremely rare [1]. The mechanism of injury remains unclear but has been attributed to shearing and/or compression forces on the extrahepatic biliary system. Morbidity rates of up to $40 \%$ have been reported, largely the result of delays in diagnosis [2-6]. Here we report a case of isolated avulsion of the hepatic duct confluence following blunt trauma that was successfully managed with Roux-en-Y hepaticojejunostomy.

\section{Case Report}

A 45-year-old man sustained blunt compression injury to his abdomen while working under a van. He was immediately extricated and presented at a referring hospital 24 hours later with mild abdominal fullness and pain. Upon transfer to our hospital, he had developed worsening abdominal pain and one episode of nonbilious emesis. He was tachycardic ( 120 beats $/ \mathrm{min}$ ) with mildly elevated blood pressure $(140 / 80)$. His abdomen was distended, diffusely tender; and without signs of external trauma. Abnormal laboratory studies included WBC $19 \times 10^{3} / \mu \mathrm{L}$, ALT 86 units/L, alkaline phosphatase 136 units/L, and total bilirubin $3.7 \mathrm{mg} / \mathrm{dL}$. Contrast-enhanced computed tomography (CT) of the chest/abdomen/pelvis revealed free fluid around the liver and right pericolic gutter (Figure 1) without evidence of extraluminal air or solid organ injury. Clips from a laparoscopic cholecystectomy (performed three years earlier for acute cholecystitis) were visualized in the hepatic fossa. Given the high suspicion for bowel and/or bile duct injury, he was taken to the operating room for exploratory laparotomy.

Exploration revealed bilious fluid that tracked into the right pericolic gutter and retroperitoneum. No injuries were detected in any solid organs or hollow viscera. Medial rotation of the right colon and duodenum did not reveal injuries in the lesser sac or retroperitoneum. The hepatic hilum was bile stained and dissection of the hepatoduodenal ligament 


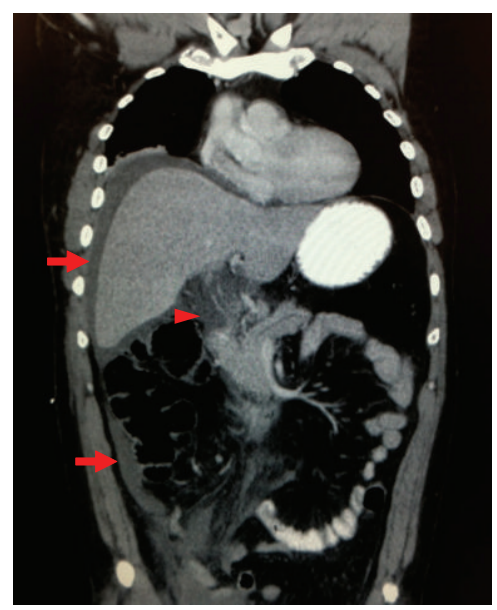

FIGURE 1: Coronal CT image demonstrating intraabdominal fluid above the liver and along the right pericolic gutter (arrows). "Periportal tracking" is also present (arrowhead), suggestive of an extrahepatic biliary injury. Possible disruption of the extrahepatic bile duct is visualized.

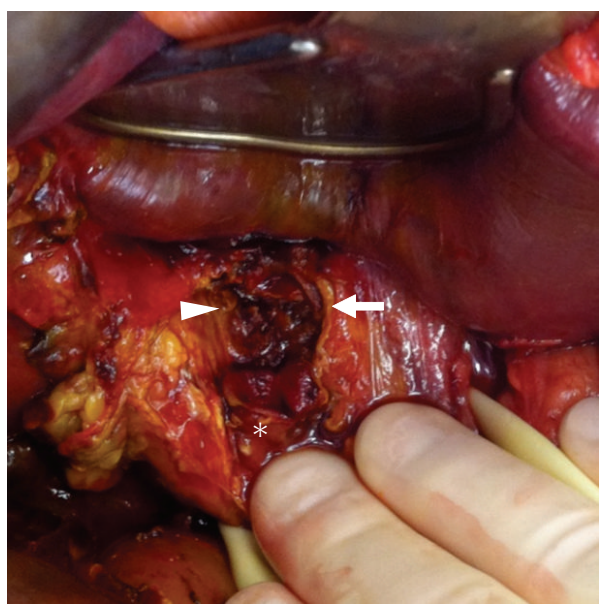

(a)

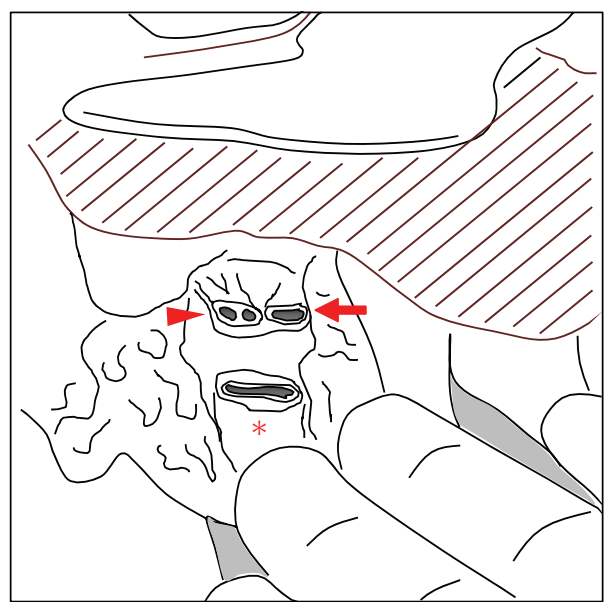

(b)

FIGURE 2: Intraoperative photograph (a) and schematic (b) demonstrating isolated avulsion of the hepatic duct confluence. The right anterior and posterior hepatic ducts (arrowhead), left hepatic duct (arrow), and common hepatic duct (asterisk) are visualized.

demonstrated complete avulsion of the hepatic duct at the confluence. The left hepatic duct and the right posterior and anterior duct junctions were visualized (Figure 2(a)). The common hepatic duct stump was oversewn and a single-layer hepaticojejunostomy was created using a retrocolic Roux-enY limb (Figure 2(b)). A stapled side-to-side jejunojejunostomy was performed and two closed suction drains were placed at the biliary-enteric anastomosis. The patient was discharged home with a single closed suction drain that was removed six weeks postoperatively. He has continued to do well at over three months after injury.

\section{Discussion}

Although isolated bile duct injuries have been described, isolated avulsion of the common hepatic duct confluence following blunt trauma has not been previously reported.
Extrahepatic biliary tract injury after blunt trauma is exceedingly rare and often associated with injuries in adjacent organs $[4,6-9]$. The diagnosis is often delayed and may be missed when the initial exploration reveals other injuries. A high index of suspicion is the key to prompt identification and treatment of extrahepatic bile duct injuries.

Several mechanisms have been proposed for blunt injury of the extrahepatic biliary system. These can be grouped into three categories: (1) crushing against the rigid spinal column, (2) shearing against areas of relative fixation, and (3) rapid emptying of a distended gallbladder into the bile ducts.

Our patient had a prior laparoscopic cholecystectomy, suggesting that rapid compression of a filled gallbladder is not an essential factor in injuries of this type. Another hypothesis proposes that shearing forces lift the liver superiorly while the hepatoduodenal ligament is pulled inferiorly [10]. The lack of concomitant arterial or venous injury in our 
patient remains puzzling but may be attributed to the relative elasticity and redundant length of the portal vein and proper hepatic artery compared to the bile ducts [1].

Diagnostic investigations commonly reported include ultrasonography and CT imaging [11]. The presence of "periportal tracking" on CT may indicate injury to the extrahepatic biliary system but is nonspecific [12]. Percutaneous aspiration may reveal bilious fluid and isotope (hepatobiliary iminodiacetic acid-HIDA) scintigraphy has been used to diagnose extrahepatic bile leaks but lacks the ability to precisely identify the injury site $[11,13]$. Magnetic resonance cholangiopancreatography (MRCP) can provide high resolution imaging of the hepatic hilum and has been used to identify biliary injury following blunt liver trauma [14]. Percutaneous transhepatic cholangiography (PTC) can also be utilized to precisely define the injury but is invasive and may be difficult to perform in nondilated bile ducts [13]. Endoscopic retrograde cholangiopancreatography (ERCP) is a valuable diagnostic option in the stable patient and is increasingly employed for the treatment of extrahepatic biliary injury [15].

Surgical options for extrahepatic ductal injuries depend on the stability of the patient [16]. In hemodynamically unstable patients, the operative goals should be external drainage and possibly primary repair for injuries involving $<50 \%$ of the duct circumference [17]. More significant injuries in an unstable patient can be treated with a bridging T-tube across the injured segments and definitive repair at a later time. Stable patients with complete transaction of an extrahepatic bile duct should undergo biliary-enteric anastomosis with a Roux-en-Y limb [18]. Primary endto-end duct repair is associated with a higher long term rate of stricture [3]. Injury to the main hepatic ducts can also be treated with a Roux-en-Y hepatoportoenterostomy, hepatic resection, or ligation of the right or left hepatic duct. Early surgical complications include infection and anastomotic leak. In the long term, stricture formation can result in recurrent cholangitis, biliary cirrhosis, and portal hypertension. Morbidity and mortality (up to $40 \%$ in some reports) are highly associated with the presence of concomitant injuries and delays in diagnosis.

To our knowledge, isolated avulsion of the hepatic duct confluence following blunt abdominal compression injury has not been previously reported. A high index of suspicion is critical to effectively identify and manage these injuries. Upon diagnosis, treatment is dictated by the hemodynamic stability of the patient and the extent of injury. Although various endoscopic, percutaneous, and surgical options are available, biliary-enteric reconstruction provides the best long term outcome for major injuries to the extrahepatic biliary system.

\section{Conflict of Interests}

The authors declare that there is no conflict of interests.

\section{Consent}

Written informed consent was obtained from the patient.

\section{References}

[1] R. W. Parks and T. Diamond, "Non-surgical trauma to the extrahepatic biliary tract," British Journal of Surgery, vol. 82, no. 10, pp. 1303-1310, 1995.

[2] M. W. Hills, A. J. Richardson, N. Tait, S. A. Deane, and J. M. Little, "Non-iatrogenic trauma to the extrahepatic biliary tract," Australian and New Zealand Journal of Surgery, vol. 63, no. 3, pp. 190-194, 1993.

[3] J. A. Rodriguez-Montes, E. Rojo, and L. García-Sancho Martín, "Complications following repair of extrahepatic bile duct injuries after blunt abdominal trauma," World Journal of Surgery, vol. 25, no. 10, pp. 1313-1316, 2001.

[4] Y. C. Chung, P. H. Lee, M. T. Huang, and C. N. Chang, "Complete transection of common bile duct due to blunt abdominal trauma: a case report and literature review," Journal of the Formosan Medical Association, vol. 92, no. 1, pp. 78-81, 1993.

[5] J. Khodadadi, M. Mihich, R. Finally, and M. Milleritzky, "Avulsion of common bile duct after blunt abdominal injury: a review of the literature," Injury, vol. 14, no. 5, pp. 447-450, 1983.

[6] M. D. Bourque, N. Spigland, A. L. Bensoussan, and L. H. Garel Blanchard, "Isolated complete transection of the common bile duct due to blunt trauma in a child, and review of the literature," Journal of Pediatric Surgery, vol. 24, no. 10, pp. 1068-1070, 1989.

[7] D. L. Dawson and G. J. Jurkovich, "Hepatic duct disruption from blunt abdominal trauma: case report and literature review," Journal of Trauma, vol. 31, no. 12, pp. 1698-1702, 1991.

[8] A. J. Shorthouse, M. P. Singh, T. Treasure, and R. H. Franklin, "Isolated complete transection of the common bile duct by blunt abdominal trauma," British Journal of Surgery, vol. 65, no. 8, pp. 543-545, 1978.

[9] B. Krishnamurthy, S. Jagdish, D. Pai, and P. Babu, "Transection of common bile duct following blunt injury to abdomen," Indian Journal of Gastroenterology, vol. 16, no. 3, pp. 109-110, 1997.

[10] L. W. C. Chow, P. Gertsch, K. L. Cheung, and S. Y. L. Leung, "Intrapancreatic avulsion of common bile duct after blunt abdominal trauma," European Journal of Surgery, vol. 162, no. 6, pp. 519-521, 1996.

[11] K. Sondenaa, A. Horn, and T. Nedrebo, "Diagnosis of blunt trauma to the gallbladder and bile ducts," European Journal of Surgery, vol. 166, no. 11, pp. 903-907, 2000.

[12] J. Yokota and T. Sugimoto, "Clinical significance of periportal tracking on computed tomographic scan in patients with blunt liver trauma," American Journal of Surgery, vol. 168, no. 3, pp. 247-250, 1994.

[13] W. L. Wahl, M. M. Brandt, M. R. Hemmila, and S. Arbabi, "Diagnosis and management of bile leaks after blunt liver injury," Surgery, vol. 138, no. 4, pp. 742-748, 2005.

[14] M. D. Kelly, C. P. Armstrong, and A. Longstaff, "Characterization of biliary injury from blunt liver trauma by MRCP: case report," Journal of Trauma, vol. 64, no. 5, pp. 1363-1365, 2008.

[15] N. P. Jaik, B. A. Hoey, and S. P. Stawicki, "Evolving role of endoscopic retrograde cholangiopancreatography in management of extrahepatic hepatic ductal injuries due to blunt trauma: diagnostic and treatment algorithms," HPB Surgery, vol. 2008, Article ID 259141, 9 pages, 2008.

[16] P. G. Bade, S. R. Thomson, A. Hirshberg, and J. V. Robbs, "Surgical options in traumatic injury to the extrahepatic 
biliary tract," British Journal of Surgery, vol. 76, no. 3, pp. 256258, 1989.

[17] V. K. Kapoor, "Management of bile duct injuries: a practical approach," American Surgeon, vol. 75, no. 12, pp. 1157-1160, 2009.

[18] M. Nathan, J. Gates, and S. J. Ferzoco, "Hepatic duct confluence injury in blunt abdominal trauma: case report and synopsis on management," Surgical Laparoscopy, Endoscopy and Percutaneous Techniques, vol. 13, no. 5, pp. 350-352, 2003. 


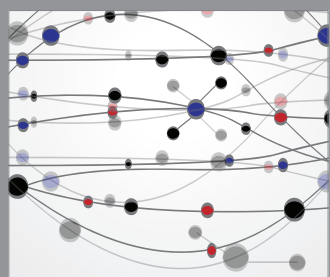

The Scientific World Journal
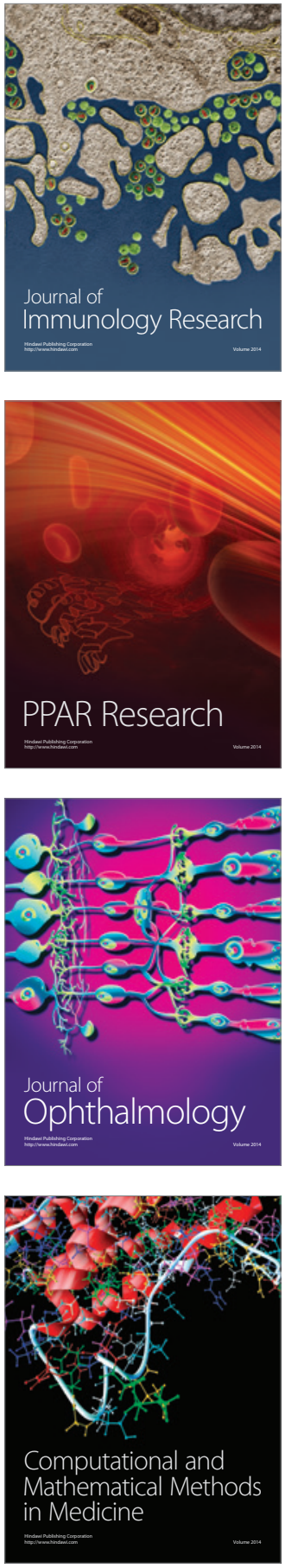

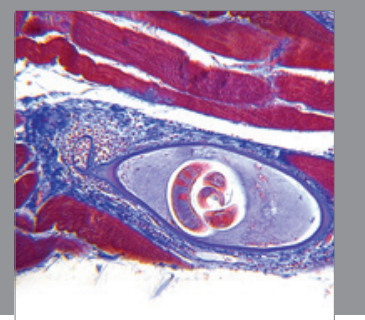

Gastroenterology

Research and Practice
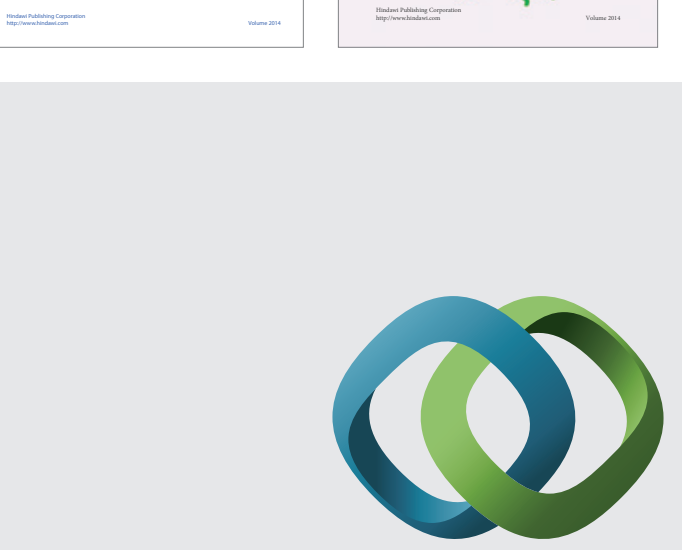

\section{Hindawi}

Submit your manuscripts at

http://www.hindawi.com
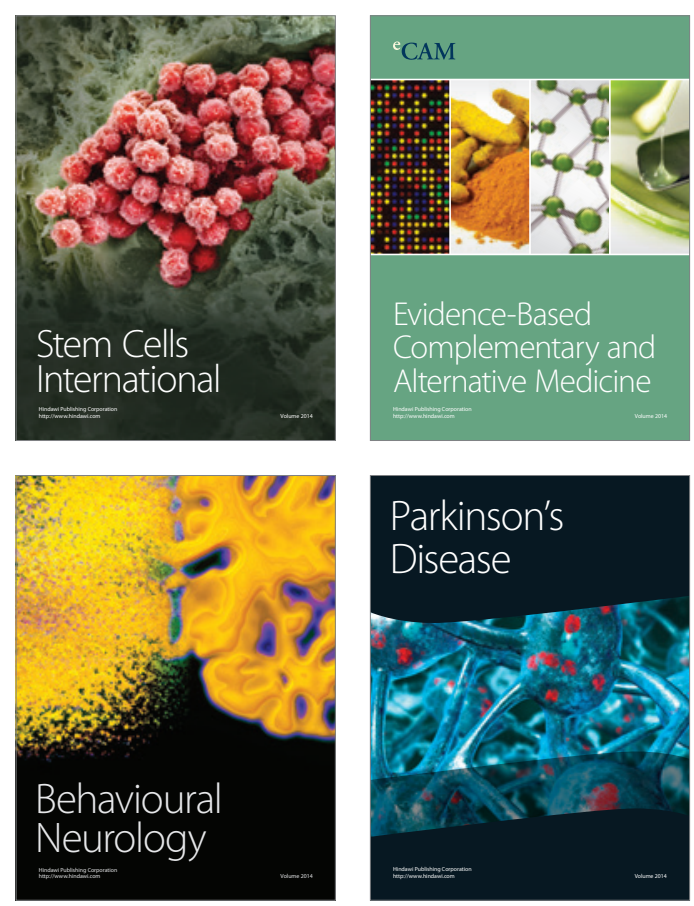

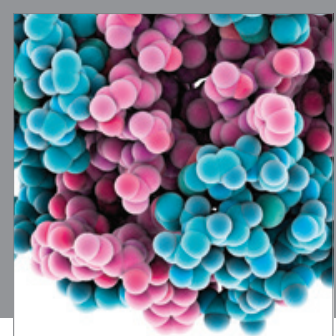

Journal of
Diabetes Research

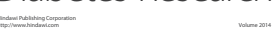

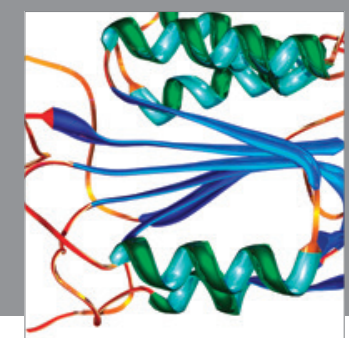

Disease Markers
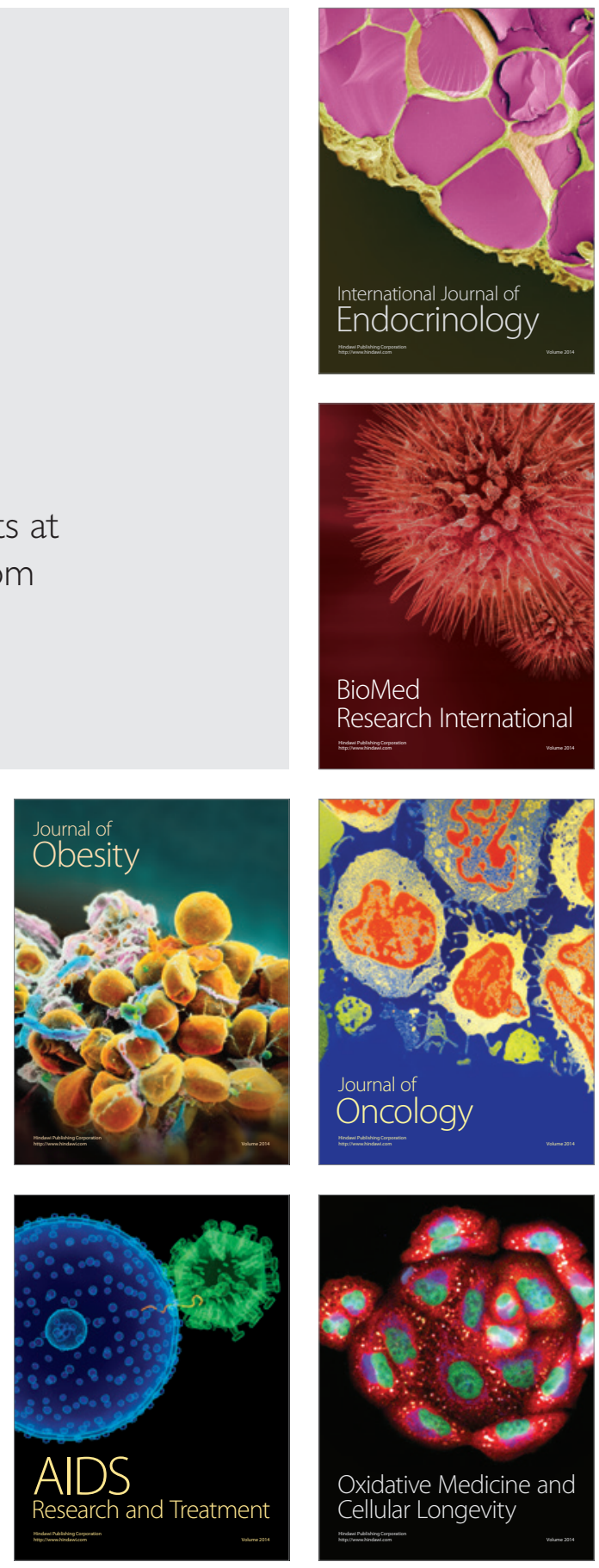